\title{
Grüneisen Ratio Divergence at the Quantum Critical Point in $\mathrm{CeCu}_{6-x} \mathrm{Ag}_{x}$
}

\author{
R. Küchler ${ }^{(1)}$, P. Gegenwart(1), K. Heuser ${ }^{(2)}$, \\ E.-W. Scheidt(2), G.R. Stewart ${ }^{(3)}$, and F. Steglich ${ }^{(1)}$ \\ (1) Max-Planck Institute for Chemical Physics of Solids, D-01187 Dresden, Germany \\ (2) EP III ,Institut für Physik, Universitätsstrasse 1, 86159 Augsburg, Germany \\ (3) University of Florida, Gainesville, FL 32611-8440, USA
}

(Dated: June 9, 2018)

\begin{abstract}
The heavy fermion system $\mathrm{CeCu}_{6-x} \mathrm{Ag}_{x}$ is studied at its antiferromagnetic quantum critical point, $x_{c}=0.2$, by low temperature $(T \geq 50 \mathrm{mK})$ specific heat, $C(T)$, and volume thermal expansion, $\beta(T)$, measurements. Whereas $C / T \propto \log \left(T_{0} / T\right)$ would be compatible with the predictions of the itinerant spin-density-wave (SDW) theory for two-dimensional critical spin fluctuations, $\beta(T) / T$ and the Grüneisen ratio, $\Gamma(T) \propto \beta / C$, diverge much weaker than expected, in strong contrast to this model. Both $C$ and $\beta$, plotted as a function of the reduced temperature $t=T / T_{0}$ with $T_{0}=4.6 \mathrm{~K}$ are similar to what was observed for $\mathrm{YbRh}_{2}\left(\mathrm{Si}_{0.95} \mathrm{Ge}_{0.05}\right)_{2}\left(T_{0}=23.3 \mathrm{~K}\right)$ indicating a striking discrepancy to the SDW prediction in both systems.
\end{abstract}

PACS numbers: 71.10.HF,71.27.+a 
The nature of the quantum critical point (QCP) at which long-range magnetic order gradually develops in heavy-fermion ( $\mathrm{HF}$ ) systems has been the focus of research activities during past years [1]. In paramagnetic systems like $\mathrm{CeCu}_{6}$, a Fermi surface formed by heavy quasiparticles has been detected, indicating an itinerant $4 f$-electron state 2]. The central question is how these heavy quasiparticles evolve if these materials are tuned into a longrange magnetically ordered state. In the traditional picture [3, 4, 5], the quasiparticles retain their itinerant character and form a spin-density wave (SDW) type of antiferromagnetic (AF) ordering. For this Hertz-Millis-Moriya theory, in the following refered as itinerant scenario, a mean-field type of quantum critical behavior arises. Recent experiments have shown that at least in some HF systems this picture fails [6, 7]. Consequently, a new type of QCP has been proposed at which the quasiparticles break-up into their components: conduction electrons and local $4 f$-moments forming magnetic order. This locally-critical scenario arises due to the destruction of the Kondo resonance at the QCP [8, 9].

In $\mathrm{CeCu}_{6}$, the weakening of the $4 f$-conduction electron hybridization, $J$, induced by a lattice expansion in $\mathrm{CeCu}_{6-x} \mathrm{M}_{x}$ with $\mathrm{M}=\mathrm{Au}\left(x_{c}=0.1\right)$ [10], $\mathrm{Ag}\left(x_{c}=0.2\right)$ [1], 12], $\mathrm{Pd}$ $\left(x_{c}=0.05\right)$ and $\mathrm{Pt}\left(x_{c}=0.1\right)$ 13] drives the system at a critical concentration $x_{c}$ towards a QCP beyond which long-range AF order appears. Remarkably, the non-Fermi liquid (NFL) behavior observed at the respective $x_{c}$ in the different systems does not depend on which dopand $\mathrm{M}$ has been used [13]. In fact, the specific heat coefficient of all of these different systems is very similar (within $\sim 10 \%$ below $3 \mathrm{~K}$ ) and shows a characteristic $C / T \propto \log \left(T_{0} / T\right)$ dependence over nearly two decades in $T$ down to $50 \mathrm{mK}$. Thus, a common origin of the quantum critical state at $x=x_{c}$ for the different systems $\mathrm{CeCu}_{6-x} \mathrm{M}_{x}$ is likely. The $\log$ arithmic divergence of the quasiparticle mass would only be compatible with the itinerant scenario if strongly anisotropic, two-dimensional (2D) critical spin fluctuations would lead to singular scattering at the whole Fermi surface [14]. Indeed, inelastic neutron scattering experiments on $\mathrm{CeCu}_{5.9} \mathrm{Au}_{0.1}$, revealed rod-like structures of high intensity in $q$-space translating to quasi-2D fluctuations in real space [15]. The 2D SDW picture, however, cannot explain that both at and far away from the wave vector of the nearby AF order the neutron and magnetization data exhibit $E / T$ and $B / T$ scaling with an anomalous exponent $\alpha<1$ 6]. The momentum independence in the critical response led to the proposal of local criticality [6, 8, 9]. So far it is not clear, which kind of mass divergence would occur in such a scenario. At the AF QCP in $\mathrm{YbRh}_{2}\left(\mathrm{Si}_{0.95} \mathrm{Ge}_{0.05}\right)_{2}$ a stronger than logarithmic mass diver- 
gence has been observed at very low temperatures, incompatible with the itinerant scenario 7]. Furthermore, local moments have been detected in the low-frequency bulk susceptibility 7], as well as in microscopic magnetic measurements [16, 17], indicating local criticality in this system. Comparing the title Ce system with this Yb system, two important questions arise: i) is there a similar discrepancy in thermodynamic properties with respect to the predictions of the itinerant scenario for the Ce system as found 7] for $\mathrm{YbRh}_{2}\left(\mathrm{Si}_{0.95} \mathrm{Ge}_{0.05}\right)_{2}$ and ii) do both materials show similar mass divergences? This Letter addresses these questions.

Very recently, it has been shown that the thermal expansion, $\beta=V^{-1}(d V / d T)(V$ : sample volume), compared to the specific heat, behavior is much more singular in the approach to the QCP. Complementary to the specific heat, being related to the temperature dependence of the entropy, the thermal expansion probes the pressure dependence of the entropy and thus should become singular in the approach of a (pressure-driven) QCP. Scaling analysis 18] revealed that the Grüneisen ratio $\Gamma \propto \beta / C$ can be used as a highly sensitive probe of quantum criticality because it has to diverge at any QCP. The critical exponent $\epsilon$ in the divergence $\Gamma \propto 1 / T^{\epsilon}$ is given by $\epsilon=1 / \nu z$ with $\nu$, the critical exponent for the correlation length, $\xi \propto|r|^{\nu}(r$ : distance from the QCP) and $z$, the dynamical critical exponent in the divergence of the correlation time, $\tau_{c} \propto \xi^{z}$ [18]. For a 3D AF QCP [19] the itinerant scenario predicts $\nu=1 / 2$ and $z=2$ yielding $\epsilon=1$. Thus a study of the Grüneisen ratio can serve as a basis for a detailed assessment of the validity of the SDW picture in different systems.

The first-ever observation of a Grüneisen ratio divergence has been made on the two HF systems $\mathrm{CeNi}_{2} \mathrm{Ge}_{2}$ and $\mathrm{YbRh}_{2}\left(\mathrm{Si}_{0.95} \mathrm{Ge}_{0.05}\right)_{2}$, both located at AF QCPs [20]. For the former system $\epsilon=1$ has been found within $50 \mathrm{mK} \leq T \leq 3 \mathrm{~K}$ in accordance with the itinerant scenario. For $\mathrm{YbRh}_{2}\left(\mathrm{Si}_{0.95} \mathrm{Ge}_{0.05}\right)_{2}$, on the other hand, the measured Grüneisen exponent is fractional: $\epsilon=0.7 \pm 0.1$ for $T \lesssim 0.8 \mathrm{~K}$ [20]. This cannot be explained by the itinerant theory, but is qualitatively consistent with the locally quantum critical picture. As this scenario has first been proposed on the basis of the neutron scattering experiments on $\mathrm{CeCu}_{6-x} \mathrm{M}_{x}(\mathrm{M}=\mathrm{Au})[6]$, it would be highly desirable, to determine the critical exponent of the Grüneisen ratio divergence in this system at the critical concentration $x_{c}$.

Below, we show first that the QCP in $\mathrm{CeCu}_{6-x} \mathrm{Ag}_{x}\left(x_{c}=0.2\right)$ is phenomenologically related to that in $\mathrm{CeCu}_{6-x} \mathrm{Au}_{x}\left(x_{c}=0.1\right)$ and afterwards investigate the nature of this QCP by means of a Grüneisen analysis. Polycrystalline samples of $\mathrm{CeCu}_{6-x} \mathrm{Ag}_{x}$ with $0.09 \leq x \leq 1.2$ were prepared via arc melting together stoichiometric amounts of high-purity elements under 
purified argon atmosphere [12, 21, 22]. They were analyzed by X-ray powder diffraction and found to be single phase and with the proper orthorhombic structure. In contrast to the Au system where for $x \leq 1$ only one of the five different $\mathrm{Cu}$ sites is occupied by the dopand, the $\mathrm{Ag}$ atoms are randomly distributed on all $\mathrm{Cu}$ sites [21]. The linear enhancement of the $b$ - and $c$-lattice parameters is observed to be of similar size as in the Au system. On the other hand, at $x=1$ the $a$-parameter enhancement is $4 \%$ larger compared to that in the $\mathrm{Au}$ system. Specific heat and thermal expansion measurements were performed in dilution refrigerators with the aid of a thermal relaxation calorimeter [12] and capacitive dilatometer 20], respectively.

Figure 1 displays the low-temperature specific heat divided by temperature, $C(T) / T$, of various $\mathrm{CeCu}_{6-x} \mathrm{Ag}_{x}$ samples on a logarithmic temperature scale. Long-range $\mathrm{AF}$ order is observed for $x \geq 0.3$ and is manifested by broadened jumps in $C(T) / T$. The inset shows $T_{N}(x)$ as determined by (entropy-conserving) equal areas constructions and the maximum of the derivative $d \rho(T) / d T$ in corresponding electrical resistivity measurements [21]. Extrapolation of $T_{N}$ to zero temperature yields a critical concentration $x_{c}=0.2$. This value is in accordance with the extrapolation of the entropy at the AF phase transition, $S\left(T_{N}\right)$, measuring the size of the ordered moment, towards zero. Thus, the critical concentration is twice as large as in the case of the $\mathrm{CeCu}_{6-x} \mathrm{Au}_{x}$ system although the relative volume expansion by Ag doping is slightly larger as in the Au case. It is unlikely that the larger value of $x_{c}$ is caused by stronger site disorder in the $\mathrm{Ag}$ system. Another possibility is that the $\mathrm{Ag}$ ions adopt a different valence state than the $\mathrm{Au}$ ions [21]. As the volume of $\mathrm{Ag}^{1+}$ ions is larger than that of $\mathrm{Au}^{2+}$ ions this would explain the relatively larger volume expansion for $\mathrm{Ag}$, compared to Au. Furthermore, a change in the electronic configuration might increase the density of states at the Fermi level. This would effectively enhance $J$ and stabilize the paramagnetic ground state leading to a higher $x_{c}$ for the Ag system [21].

For the $\mathrm{CeCu}_{5.8} \mathrm{Ag}_{0.2}$ sample which is located right at the QCP, the specific heat coefficient is very similar to that in $\mathrm{CeCu}_{5.9} \mathrm{Au}_{0.1}$ and, at zero magnetic field, diverges logarithmically between $50 \mathrm{mK}$ and $2.5 \mathrm{~K}$ (Figure 2a). The application of magnetic fields leads to the recovery of Landau Fermi-liquid behavior, $C / T=\gamma(B)$, at lowest temperatures as observed in many other NFL systems [1]. We observe a logarithmic divergence $\gamma(B) \propto-\log B$ (not shown) which represents a clear signature of a QCP at $B=0$. The zero-field temperature dependence, as well as the field dependence of $\gamma(B)$, assuming a linear relation between the 
magnetic field and the control parameter, would be compatible with the itinerant theory for an AF QCP, if one assumes truely 2D critical spin fluctuations.

We now turn to the thermal expansion, measured along three perpendicular orientations on the same polycrystal studied by specific heat (Figure 2b). The observed anisotropy is caused by the texture of the polycrystalline sample. The volume expansion coefficient $\beta$ is given by the sum of the three linear expansion coefficients $\alpha_{i}$ all showing a similar temperature dependence. Upon cooling to the lowest temperatures, $\beta(T) / T$ increases strongly and diverges logarithmically for $T \leq 0.8 \mathrm{~K}$. Although the observed $\beta / T$ divergence is steeper than in $C / T$, indicating that the NFL behavior is caused by a QCP, it is much weaker than the temperature dependence expected in the 2D-SDW scenario, $\beta(T) / T=a_{0}+a_{1} / T+a_{2} / T \log \log \left(T_{0} / T\right)[18]$.

Figure 3 displays the temperature dependence of the Grüneisen ratio $\Gamma(T)$ calculated from the specific heat and thermal expansion data shown in Figure 2. In the entire temperature range, the divergence is weaker than $\Gamma \propto 1 / T$ and thus incompatible with the predictions of the itinerant scenario for both 3D or 2D critical spin fluctuations [18]. As shown in the inset, $\Gamma(T)$ roughly follows a logarithmic increase upon cooling from below $1 \mathrm{~K}$.

In the last part of this paper, we compare the NFL behavior in $\mathrm{CeCu}_{5.8} \mathrm{Ag}_{0.2}$ with that observed in $\mathrm{YbRh}_{2}\left(\mathrm{Si}_{0.95} \mathrm{Ge}_{0.05}\right)_{2}$ [7, 20]. As discussed by Sereni et al. 24] for a substantial number of HF systems located near the magnetic instability the logarithmic increase of the specific heat coefficient $C / T$ involves a similar amount of entropy (about $55 \%$ of $R \ln 2$ ). On a reduced temperature scale $t=T / T_{0}$, where the spin-fluctuation temperature $T_{0}$ is assumed to be close to the Kondo temperature $T_{K}$ determined, e.g. from the residual quasielastic neutron line width, the specific heat coefficient is described by $C / t=-D \log t+$ $E T_{0}$ with $D=7.2 \mathrm{Jmol}^{-1} \mathrm{~K}^{-1}$ [24]. Here the parameter $E$ is small or negligible for systems showing a low Kondo temperature as well as excited crystal electric field states that are well separated from the ground state. In Figure 4, we show that our specific heat data agree perfectly with the scaling function using $T_{0}=4.6 \mathrm{~K}$ (see straight line). Similar scaling behavior has been reported for the $\mathrm{CeCu}_{6-x} \mathrm{Au}_{x}$ series as well 24]. The specific heat data of $\mathrm{YbRh}_{2}\left(\mathrm{Si}_{0.95} \mathrm{Ge}_{0.05}\right)_{2}$ collapse for $t \geq 0.01$ on the scaling function with $T_{0}=23.3 \mathrm{~K}$ (see Figure 4). Indeed, this value agrees well with the single-ion Kondo temperature $T_{K} \approx 25 \mathrm{~K}$ for this system [7]. For $t<0.01$ the specific heat coefficient of $\mathrm{YbRh}_{2}\left(\mathrm{Si}_{0.95} \mathrm{Ge}_{0.05}\right)_{2}$ shows a pronounced upturn that is not a precursor of the tiny $\mathrm{AF}$ order at $T_{N}=20 \mathrm{mK}$ [7]. Above 
$50 \mathrm{mK}$, the zero-field data of both specific heat and thermal expansion are identical to their counterparts at the critical field $B_{c}=0.027 \mathrm{~T}$ [20]. Since the stronger than logarithmic mass divergence in this compound is at strong variance to the predictions of the itinerant model, it would be highly desirable to probe the specific heat of the Ce-based systems at $t \leq 0.01$. For $\mathrm{CeCu}_{5.8} \mathrm{Ag}_{0.2}$, this corresponds to the temperature range below $50 \mathrm{mK}$ which has, unfortunately, not been investigated so far.

Finally, we turn to the scaling behavior observed in the thermal expansion. In contrast to the specific heat, the thermal expansion of $\mathrm{CeCu}_{5.8} \mathrm{Ag}_{0.2}$ deviates strongly from the predictions of the itinerant theory for $T \geq 50 \mathrm{mK}$. In Figure 5 we demonstrate that, using the same $T_{0}$ values obtained from the specific heat scaling, the thermal expansion shows scaling behavior for both systems as well. As mentioned before, the SDW theory in the 2D case would predict a very strong divergence $\beta / T \propto T^{-1} \log \log T$. By contrast, a logarithmic increase is observed upon cooling over more than one decade in $t$ for $0.015 \lesssim t \lesssim 0.25$, followed by a $1 / t$ divergence in the $\mathrm{Yb}$ system. Already the weak logarithmic divergence above $0.015 t$ rules out the 2D SDW scenario for the QCP in both systems.

In summary, the thermodynamic behavior in $\mathrm{CeCu}_{5.8} \mathrm{Ag}_{0.2}$, which is located right at an AF QCP, has been studied by measurements of the specific heat and the thermal expansion. The observed logarithmic temperature dependence of $C / T$ would be compatible with the itinerant theory assuming the presence of 2D critical spinfluctuations. Such a scenario, can however clearly be excluded from the analysis of the thermal expansion and Grüneisen ratio data. Both properties diverge towards zero temperature much weaker than predicted by the itinerant theory. On the reduced temperature scale $T / T_{0}$, with $T_{0} \approx T_{K}$, the Kondo temperature, one finds universal behavior in the thermodynamic properties of both $\mathrm{CeCu}_{5.8} \mathrm{Ag}_{0.2}$ and $\mathrm{YbRh}_{2}\left(\mathrm{Si}_{0.95} \mathrm{Ge}_{0.05}\right)_{2}$ for which latter material a locally critical QCP had been highlighted recently [7, 17, 20].

We are grateful to C. Geibel, J. Sereni and Q. Si for stimulating discussions. Part of the work at Dresden was supported by the Fonds der Chemischen Industrie (Dresden). Work at Augsburg was supported by the Deutsche Forschungsgemeinschaft (SFB 484).

[1] G.R. Stewart, Rev. Mod. Phys. 73, 797 (2001) and references therein.

[2] P.H.P. Reinders, et al., Phys. Rev. Lett. 57, 1631 (1986). 
[3] J.A. Hertz, Phs. Rev. B 14, 1165 (1976).

[4] A.J. Millis, Phys. Rev.B 48, 7183 (1993).

[5] T. Moriya and T. Takimoto, J. Phys. Soc. Jpn. 64, 960 (1995).

[6] A. Schröder et al., Nature 407, 351 (2000).

[7] J. Custers et al., Nature 424, 524 (2003).

[8] Q. Si et al., Nature 413, 804 (2001).

[9] P. Coleman et al., J. Phys. Condens. Matter 13, R723 (2001).

[10] H. von Löhneysen et al., Phys. Rev. Lett. 72, 3262 (1994).

[11] G. Fraunberger, et al., Phys. Rev. B 40,4735 (1989).

[12] K. Heuser et al., Phys. Rev. B 57, R4198 (1998).

[13] M. Sieck et al., Physica B 223\&224, 325 (1996).

[14] A. Rosch et al., Phys. Rev. Lett. 79, 159 (1997).

[15] O. Stockert et al., Phys. Rev. Lett. 80, 5627 (1998).

[16] K. Ishida et al., Phys. Rev. Lett. 89, 107202 (2002).

[17] J. Sichelschmidt et al., Phys. Rev. Lett. 91, 156401 (2003).

[18] L. Zhu et al., Phys. Rev. Lett. 91, 066404 (2003).

[19] For 2D critical spin fluctuations an even stronger divergence $\Gamma \propto \log \log T / T \log T$ is predicted [18].

[20] R. Küchler et al., Phys. Rev. Lett. 91, 066405 (2003).

[21] K. Heuser, Dissertation, Universtität Augsburg, 1999.

[22] E.-W. Scheidt, et al., Physica B 321, 133 (2002).

[23] G. Oomi et al., J. Phys. Soc. Jpn. 57, 152 (1998).

[24] J. Sereni, Physica B 230-232, 580 (1997). 


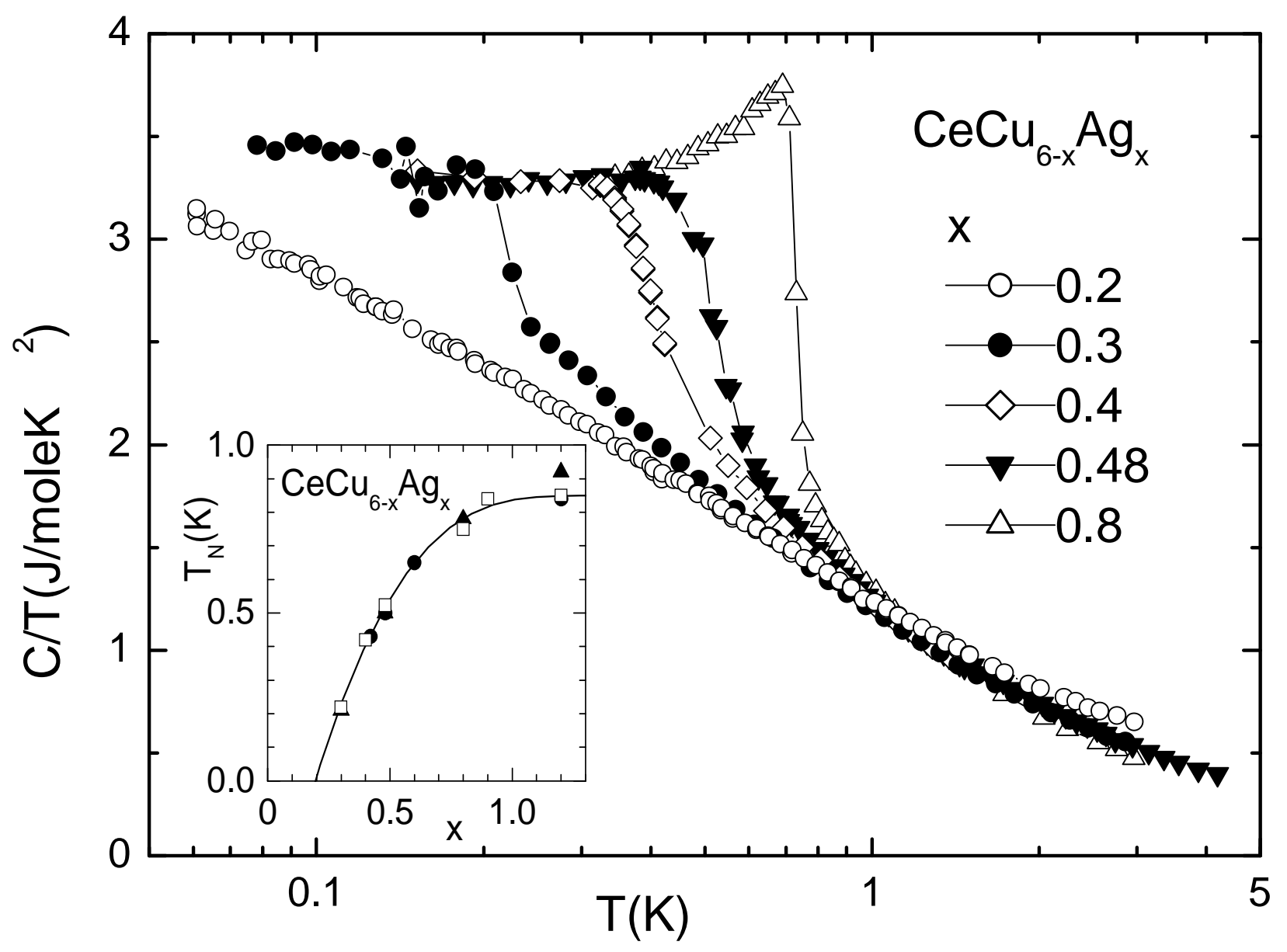

FIG. 1: Specific heat as $C / T$ vs $T$ (on a logarithmic scale) for different $\mathrm{CeCu}_{6-x} \mathrm{Ag}_{x}$ polycrystals. Inset shows evolution of antiferromagnetic phase transition temperature $T_{N}$ vs $x$ as derived from specific heat (squares: this study, circles [1]]) and electrical resistivity (triangles [21]) results. 


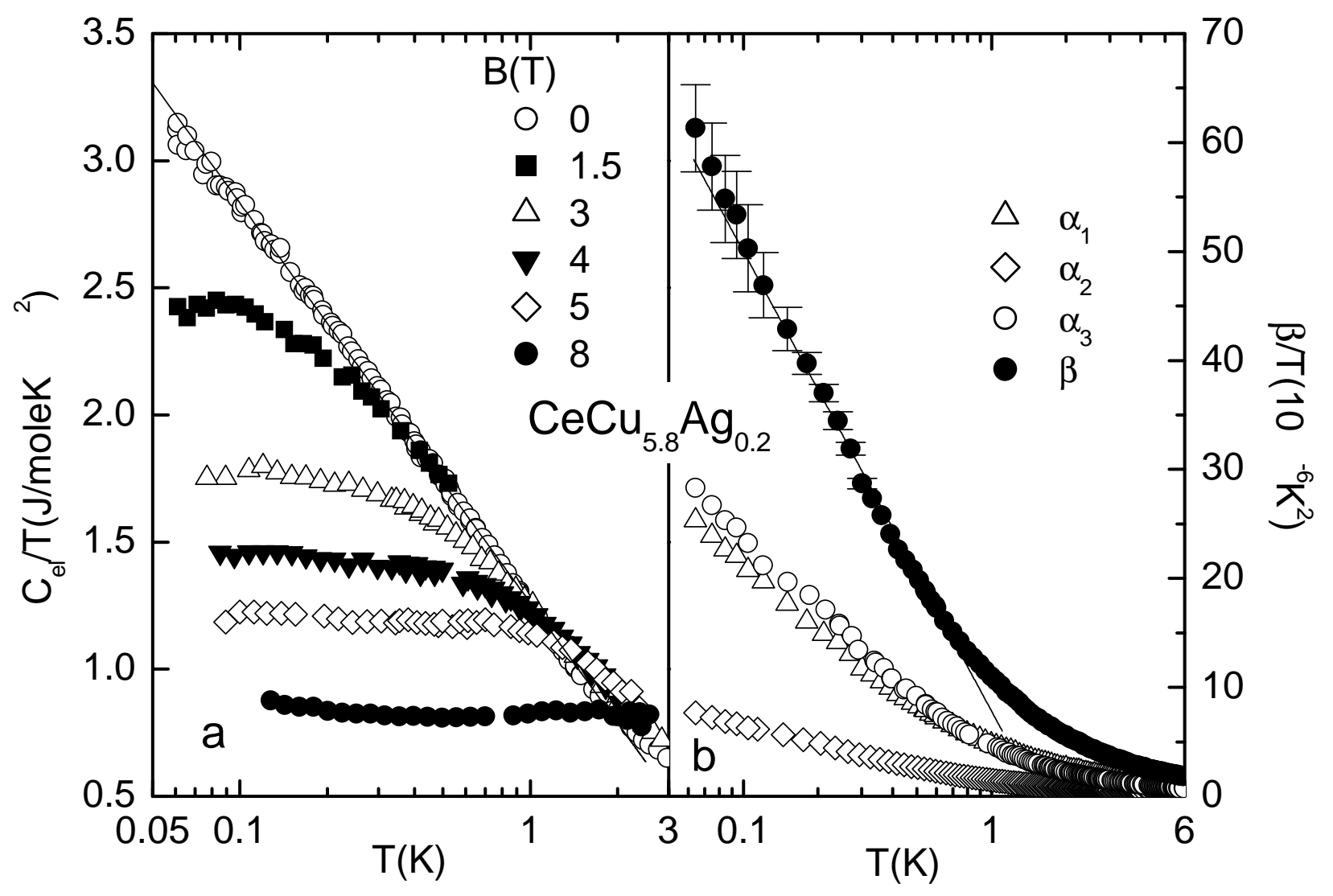

FIG. 2: (a) Electronic specific heat of $\mathrm{CeCu}_{5.8} \mathrm{Ag}_{0.2}$ as $C_{e l} / T$ vs $T$ (on a logarithmic scale) for $B=0$ and differing magnetic fields. At $B>0, C_{e l}$ is obtained after subtraction of the $\mathrm{Cu}$ nuclear specific heat contribution $C_{n} \propto B^{2} / T^{2}[21]$. (b) Volume thermal expansion coefficient $\beta$ of the same sample studied in (a) as $\beta / T$ vs $\log T$. $\beta=\alpha_{1}+\alpha_{2}+\alpha_{3}$ with $\alpha_{i}$ being the linear thermal expansion coefficients along the three perpendicular directions of the sample. Solid lines indicate logarithmic temperature dependences in $C_{e l}(T) / T$ and $\beta(T) / T$. 


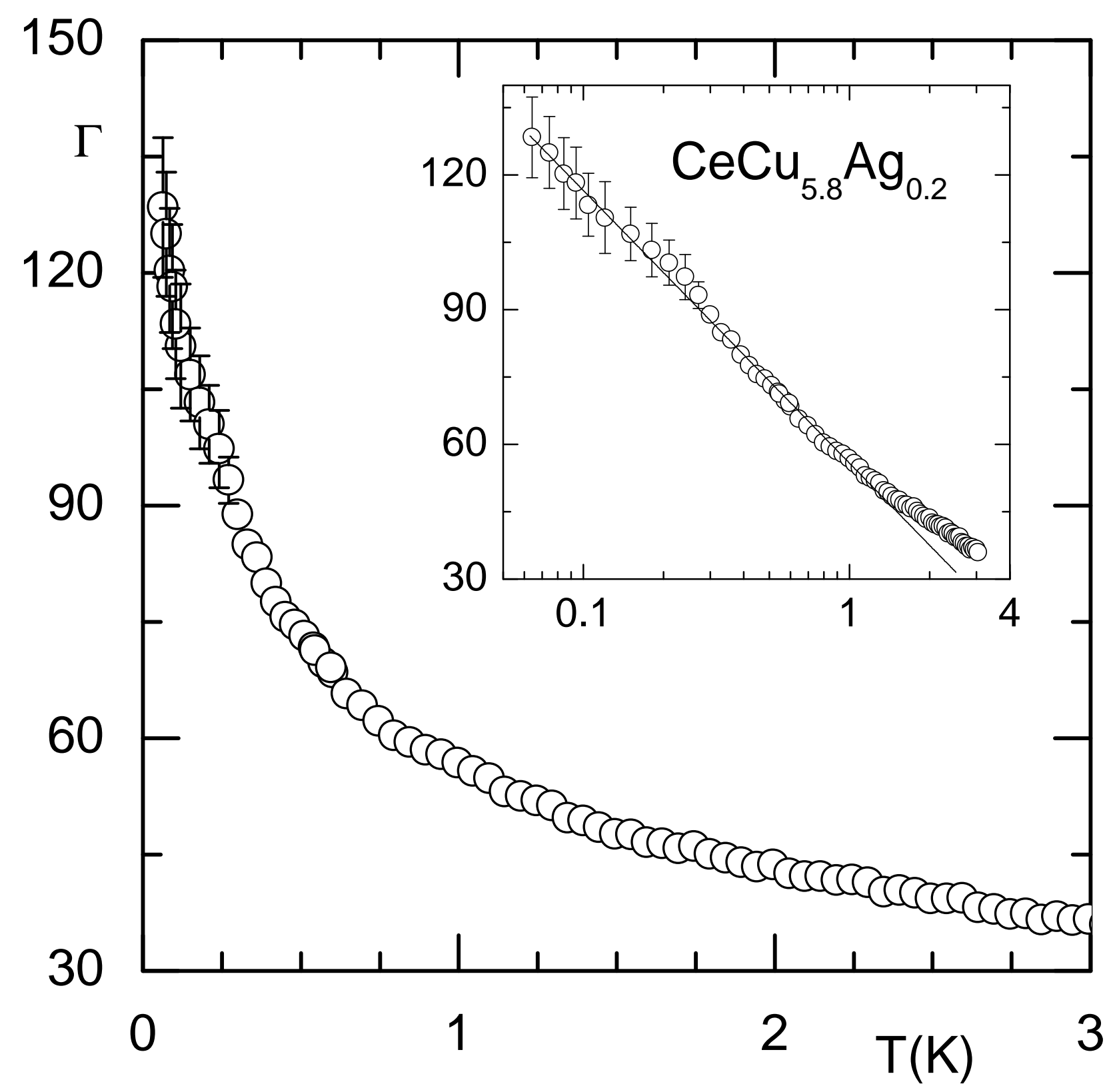

FIG. 3: Temperature dependence of Grüneisen-ratio $\Gamma=V_{m o l} / \kappa_{T} \cdot \beta / C$ with molar volume $V_{m o l}=$ $6.37 \cdot 10^{-5} \mathrm{~m}^{3} \mathrm{~mol}^{-1}$ and isothermal compressibility $\kappa_{T}=1 \cdot 10^{-11} \mathrm{~Pa}^{-1}$ [23]. Inset shows same data on a logarithmic temperature scale. Solid line represents $-\log (T)$ dependence. 


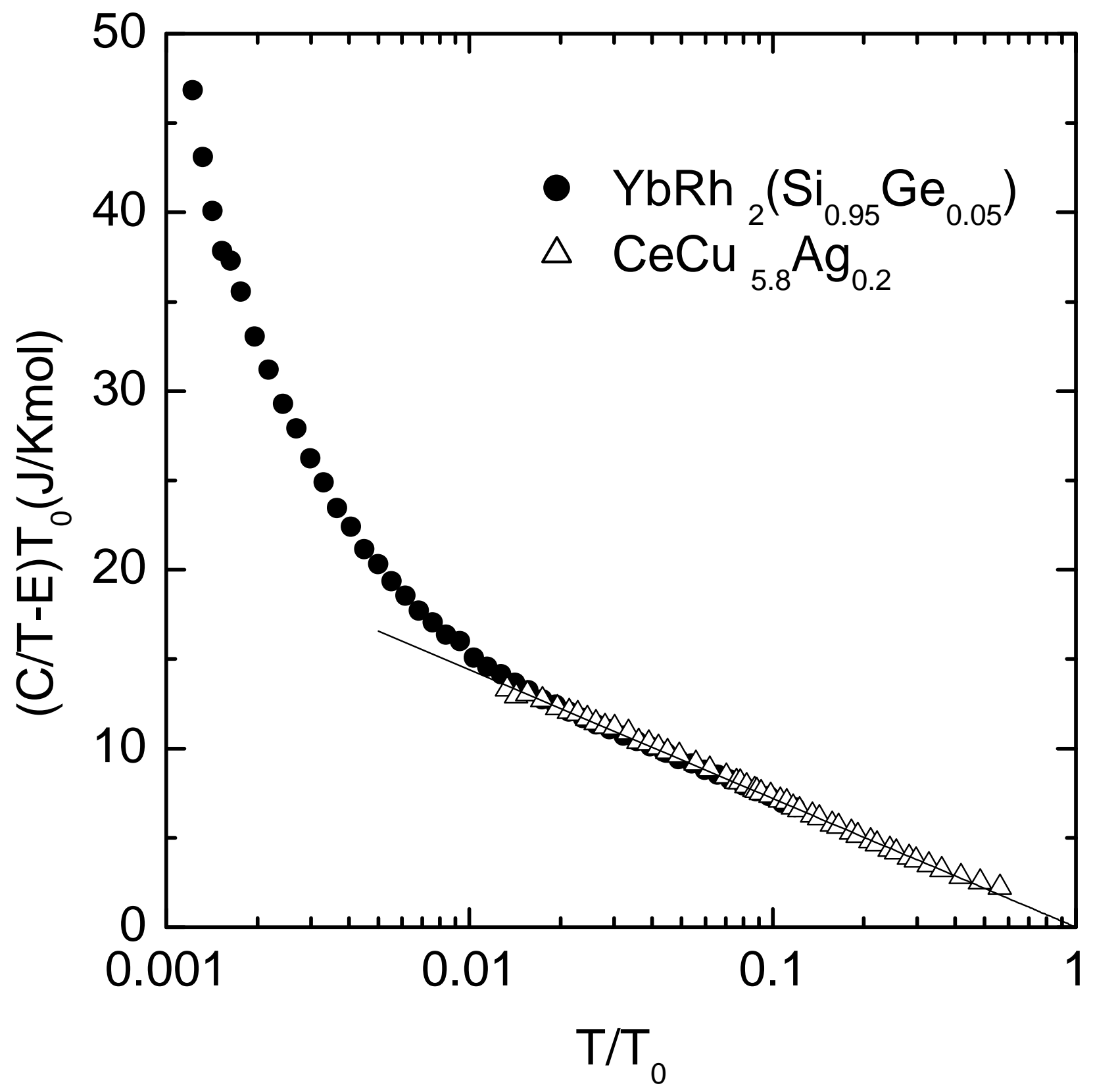

FIG. 4: Scaling of the low- $T$ specific heat of $\mathrm{CeCu}_{5.8} \mathrm{Ag}_{0.2}$ and $\mathrm{YbRh}_{2}\left(\mathrm{Si}_{0.95} \mathrm{Ge}_{0.05}\right)_{2}$ [7, 20] as $(C / T-E) T_{0}$ vs $\log \left(T / T_{0}\right)$ according to Sereni et al. [24]. For $\mathrm{CeCu}_{5.8} \mathrm{Ag}_{0.2}, T_{0}=4.6 \mathrm{~K}$ and $E=0.105 \mathrm{~J} / \mathrm{K}^{2} \mathrm{~mol}$, for $\mathrm{YbRh}_{2}\left(\mathrm{Si}_{0.95} \mathrm{Ge}_{0.05}\right)_{2}, T_{0}=23.3 \mathrm{~K}$ and $E=0.066 \mathrm{~J} / \mathrm{K}^{2} \mathrm{~mol}$. Solid line represents scaling function $7.2 \log \left(T_{0} / T\right)$ observed in several other HF systems [24]. 


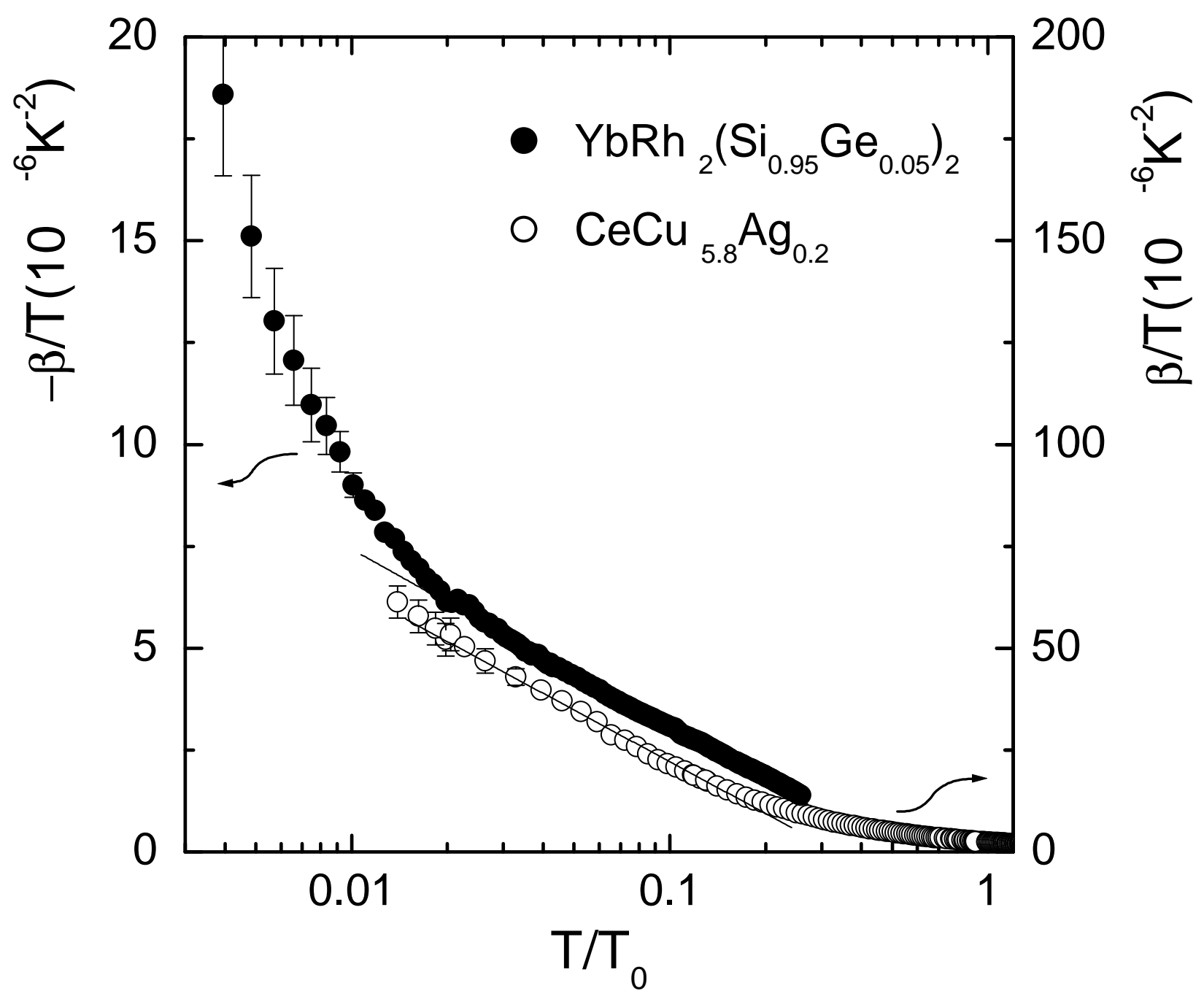

FIG. 5: Volume thermal expansion as $\beta / T$ vs normalized temperature $T / T_{0}$ (on a logarithmic scale) for $\mathrm{CeCu}_{5.8} \mathrm{Ag}_{0.2}\left(T_{0}=4.6 \mathrm{~K}\right.$, right axis $)$ and $\mathrm{YbRh}_{2}\left(\mathrm{Si}_{0.95} \mathrm{Ge}_{0.05}\right)_{2}[20]\left(T_{0}=23.3 \mathrm{~K}\right.$, left axis). Parallel solid lines indicate logarithmic temperature dependence of $\beta(T) / T$. 